\title{
Zum Körpergrab der Mitteldonauländischen Hügelgräberkultur von Straning, Niederösterreich
}

\author{
On a grave of the Middle Danube Tumulus Culture \\ from Straning, Lower Austria
}

\section{Zoja Benkovsky-Pivovarová}

\begin{abstract}
Abstrakt
Aus einem rezent gestörten Körpergrab einer in rechter Hockerlage bestatteten anzunehmenden Frau stammen eine Nadel, fünf Herzanhänger, sieben Tutuli und ein Krug mit senkrechter Rillenverzierung. Der Krug datiert dieses Grab in die jüngere Stufe der Mitteldonauländischen Hügelgräberkultur und bezeugt die Lebensdauer der in den Gräbern der älteren und mittleren Stufe der mittleren Bronzezeit von Großhöflein, Hollabrunn und Pitten in Ostösterreich vertretenen Typen der Herzanhänger bis in ihre jüngere Stufe. Die Untersuchungen zur Bestattung der Toten in der Hockerlage in der Mitteldonauländischen Hügelgräberkultur deuten die Möglichkeit der Beisetzung der Männer auf der linken und der Frauen auf der rechten Seite an, bei der gegenwärtigen Quellenvorlage lässt es sich aber nicht verifizieren.
\end{abstract}

\section{Schlüsselwörter}

Niederösterreich, Körpergrab, rechte Hockerlage, Herzanhänger, rillenverzierte Keramik, jüngere Phase der Mitteldanubischen Hügelgräberkultur.

\begin{abstract}
Abstrakt
Z recentne narušeného kostrového hrobu pochádza ihlica, pät’ srdcovitých záveskov, sedem puklíc a džbánok. Zvislo žliabkovaný džbánok datuje tento hrob do mladšieho stupňa stredodunajskej mohylovej kultúry a dokazuje výskyt takmer všetkých typov srdcovitých záveskov z hrobov staršieho a stredného stupňa strednej doby bronzovej z Großhöfleinu, Hollabrunnu a Pittenu vo východnom Rakúsku až do jej mladšieho stupňa. Poznatky k pochovávaniu v skrčenej polohe v stredodunajskej mohylovej kultúre naznačujú možnost' pochovávania mužov na l'avom a žien na pravom boku, za súčasného stavu publikovania prameňov sa to však nedá verifikovat'.
\end{abstract}

\section{Klíčová slova}

Dolní Rakousko, kostrový hrob, uložení na pravém boku, srdcovité závěsky, žlábkovaná keramika, mladši fáze středodunajské mohylové kultury. 
Im Jahre 1957 wurde vom damaligen Kustos des Höbarth-Museums in Eggenburg Franz Schäffer das Körpergrab von Straning im nördlichen Niederösterreich veröffentlicht (Schäffer 1957), welches trotz seines wichtigen Inventars in der Literatur weitgehend unbemerkt blieb (Zeichnungen übernommen von Maurer 1989, Abb. 22). Dieser Beitrag stützt sich auf die erwähnte Erstpublikation und den Fundbericht F. Schäffers, der mir in dankenswerter Weise vom Direktor des Krahuletz-Museums in Eggenburg Dr. Johannes M. Tuzar zur Verfügung gestellt wurde. Wegen der eingeschränkten Arbeitsmöglichkeiten im Jahre 2020 wurden die Zeichnungen F. Schäffers nur umgezeichnet.

Auf das Grab stieß man in einem Weingarten auf der Parzelle Nr. 95 bei der Erweiterung der Straße Straning-Grafenberg. Die Arbeiter brachten fünf gefundene Tutuli dem Schulleiter von Straning, der das Krahuletz-Museum in Eggenburg verständigte. Vom Museum wurde sofort eine Notgrabung durchgeführt, bei der sich herausstellte, dass dieses Grab bereits in der Vergangenheit beim Einpflanzen eines Weinstocks angeschnitten wurde. Trotzdem blieben die Ostwand und ein Teil der Südwand der Grabgrube erhalten und Teile des Skelettes und des Inventars wurden noch in ursprünglicher Lage angetroffen. Im durchsuchten Aushub kamen keine Funde zum Vorschein (Schäffer 1957, 1).

Vom Skelett blieben der Schädel sowie die Knochen des rechten Armes und des rechten Beines erhalten; die Arm- und Beinknochen befanden sich in rechter Hockerlage und beim Schädel wurde die Blickrichtung nach Osten festgehalten, sodass eine S-W Orientierung des/r Toten wahrscheinlich ist. Aufgrund der erhaltenen Skelettreste nahm F. Schäffer an, dass in diesem Grab eine jugendliche Frau beigesetzt war (Schäffer 1957, 1).

Die Lage der bereits gehobenen Nadel ließ sich nicht mehr feststellen. Im Halsbereich wa- ren fünf herzförmige Anhänger verstreut, die durch Bronzepatina verfärbten Knochen des rechten Unterarmes deuten die Existenz vom Armschmuck an, beim rechten Knie fanden sich zwei Tutuli, mit gewölbter Seite nach oben auf dem Ober- und Unterschenkel liegend, weswegen der Ausgräber an ihre Funktion als Bekleidungsschmuck dachte (Schäffer 1957, 3), südlich des Schädels, etwa $15 \mathrm{~cm}$ vom Schädeldach entfernt, lag ein zerdrücktes Gefäß.

Die Rollenkopfnadel mit glattem Schaft (Abb. 1: 1) ist als ein langlebiger Nadeltyp für die Datierung dieses Grabes irrelevant (z. B. Novotná 1980, Taf. 66), deswegen sei lediglich das Vorkommen von zwei Rollenkopfnadeln im Körpergrab 48 von Smolenice in Begleitung einer kleinen Amphore mit senkrechter Rillenverzierung erwähnt (Dušek 1980, Taf. VI: 10, 11, 15); angesichts der herzförmigen Anhänger ist anzunehmen, dass auch das Grab von Straning ursprünglich zwei Rollenkopfnadeln enthielt. Ebenfalls die kalottenförmigen Tutuli sind für eine nähere Datierung des Grabes ungeeignet. Die unverzierten und verzierten Tutuli (Abb.1: 7-13) waren im Arbeitsgebiet seit der frühen Bronzezeit im Gebrauch; mit ihnen befassten sich in der jüngsten Zeit mehrere Autoren (Hlas - Stuchlik - Š́n 2017, 63 f.; Bartik - Farkaš - Jelinek 2019, 76, Taf. 15). Stellvertretend für die Tutuli der Mitteldonauländischen Hügelgräberkultur (im Folgenden: MHK) seien jene aus den Gräbern von Nymburg, Čáčov und Šamorín genannt (Beneš 1959, 42, Taf. 15; Novotná 1980, Taf. 56G; Godiš - Haruštiak 2020, Taf. III). Der große verzierte Tutulus von Straning (Abb. 1: 12) repräsentiert den Typ C der Tutuli der MHK in Mittelböhmen (Beneš 1959, 20).

Die Herzanhänger, von H. Müller-Karpe als „Hörnerpaar-Anhänger“ interpretiert (Müller-Karpe 2003, 147-150; weitere symbolische Deutungen: Koschik 1981, 93), wurden nur von Frauen getragen, und zwar meistens als Halsschmuck (z. B. Schumacher-Matthäus 1985, 105). 
Ihr Herkunftsgebiet war das Karpatenbecken, aber mit einer Verspätung gelangten sie bis nach Bayern, wobei in den einzelnen Regionen ihres Verbreitungsgebietes auch lokale Typen entstanden sind (z. B. Mozsolics 1967, 87-89; Hänsel 1968, 115-118, Verbreitungskarten 25 und 26; Furmánek 1973, 125; Wells-Weyrauch 1991, 34, Verbreitungskarten auf S. 38-40). In der MHK bestanden die Halsketten entweder aus Anhängern vom selben Typ oder aus verschiedenartigen Anhängern (Willvonseder 1937, 308, Taf. 33: 1-3; Dušek 1980, Taf. IV: 16-27; VII: 13-18; Lauermann 1991, Taf. 4: 9-12; Godiš - Haruštiak 2020, Taf. III). Die fünf Anhänger von Straning gehören vier Grundtypen an; einer von ihnen ist in zwei Varianten vorhanden. Die Aufhängezunge der Anhänger ist ungleich lang und immer zu einer Öse eingerollt, bei einem Anhänger wurde die Gussnaht nicht entfernt (Abb. 1: 3). Da für die Datierung des Grabes das Gefäß entscheidend ist, werden als Analogien der Anhänger ausschließlich die Exemplare aus verlässlich bronzedatierten Grabinventaren in Ostösterreich genannt. Als Orientierungshilfe sind bei den einzelnen Anhängertypen ohne Anspruch auf Vollständigkeit die in der Literatur für sie verwendeten Termini inklusive der Datierung angeführt.

Typ 1 (Abb. 1: 2). Herzanhänger mit einfachem Mittelsteg (Variante 7 der umgekehrt herzförmigen Anhänger, MD III-SD: Hänsel 1968, 117 f.; Typ Matejovce, mittlere Hügelgräberzeit: Furmánek 1980, 29 f.; Typ Schwaighauser Forst, mittlere und jüngere Hügelgräberzeit: Wels-Weyrauch 1991, 38-40). Dieser Anhängertyp ist mit vier Exemplaren im Bronzeinventar eines Doppelgrabes eines Mannes und einer Frau von Hollabrunn vertreten, das u. a. einen zweinietigen Dolch, drei Pfeilspitzen, eine Petschaftkopfnadel, einen Drahtarmring und eine Pinzette enthielt; von E. Lauermann wurde dieses Grab in die Stufe B2/C1 datiert (Lauermann 1991, 37, Taf. 4: 9-12 mit älterer Literatur).

Typ 2 (Abb. 1: 3, 4). Herzanhänger mit Mittelsteg und kurzen Fortsätzen (Variante 6 der umgekehrt herzförmigen Anhänger, MD II-MD III: Hänsel 1968, 117; Typ Dunajská Streda, mittlere Hügelgräberzeit: Furmánek 1980, 30; Typ Oberbernbach, mittlere und jüngere Hügelgräberzeit: Wels-Weyrauch 1991, 43-47). Diesen Anhängern steht der Anhänger aus dem Kindergrab 26a von Pitten nahe, der aufgrund seiner Vergesellschaftung mit einer rundschaftigen Lochhalsnadel und einer Petschaftkopfnadel in die mittlere Stufe der Hügelgräberkultur datiert wurde (Hampl et al. 1981, Taf. 203: 6-8; Benkovsky-Pivovarová 1985, 72, 96 f.).

Typ 3 (Abb. 1: 5). Herzanhänger mit verzweigtem Mittelsteg und kurzen Fortsätzen (Herzanhänger mit verzweigtem Mittelsteg, Koszider Horizont und mittlere Hügelgräberzeit: Furmánek 1980, 27 f.; Typ Aying, mittlere und jüngere Hügelgräberzeit: Wels-Weyrauch 1991, 47-49). Im Grab Fst. 42 von Großhöflein ist dieser Anhängertyp durch zwei Sichelnadeln in die Stufe B1 datiert (Willvonseder 1937, Taf. 24: 2-4).

Typ 4 (Abb. 1: 6). Zum Herzanhänger mit kurzem, verzweigtem, eckigem Mittelsteg sind m. W. in Ostösterreich keine Parallelen bekannt; er ist am ehesten mit dem südbayerischen Typ Appenstetten vergleichbar (mittlere und jüngere Hügelgräberzeit: Wels-Weyrauch 1991, 49 f., insb. Taf. 15: 377a).

Der rillenverzierte Krug (Abb. 1: 14) stellt eine typische Form der jüngeren Stufe der MHK dar (Stuchlik 1993, 285). Mit der Rillenverzierung der Keramik dieser Kultur befasste sich eingehend J. Říhovský in seiner Arbeit über die Grundlagen der Mitteldonauländischen Urnenfelderkultur in Mähren; er zeigte, dass 



$1-13$
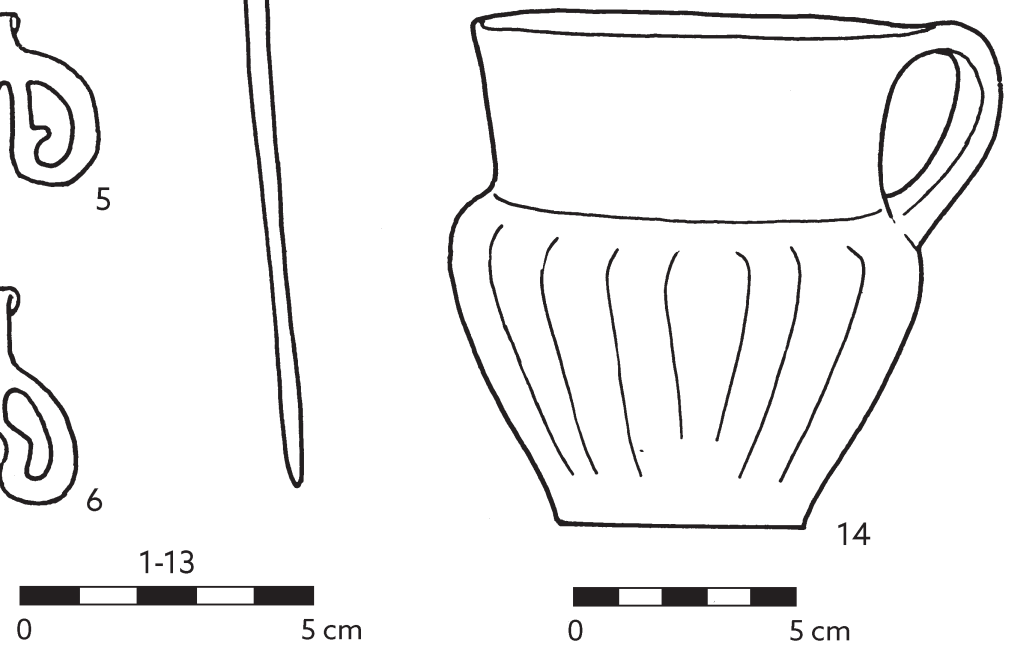

Abb. 1: Straning, Inventar des Körpergrabes der Mitteldonauländischen Hügelgräberkultur nach F. Schäffer (Umzeichnung S. Plchová; M = Bronzen 2:3, Krug 1:2).

Obr. 1. Straning, inventár kostrového hrobu stredodunajskej mohylovej kultúry podla F. Schäffera (Překreslila S. Plchová; $M$ = bronzy 2:3, dzbánok 1:2). 
sie während der jüngeren und späten Phase der MHK bei der Verzierung von Amphoren, Krügen und amphorenartigen Schüsseln angewendet wurde (Ǩihovský 1982, 19 f.). Der Autor unterschied dabei drei Varianten der Rillenverzierung:

1) Breite und seichte, meistens senkrechte, durch Seitendruck geformte Rillen, die glatte, wenig ausgeprägte Rippen entstehen lieBen (̌̌ihouský 1982, 19, Taf. 2: 1, 6, 7).

2) Klassische Variante - senkrechte aneinandergereihte Rillen (Ǩihouský 1982, 20, Taf. 2: 4, 5, 12).

3) Senkrechte schmale Rillen bzw. breitere, seichte Ritzlinien, die durch glatte Flächen voneinander getrennt sind (Řihovský 1982, 20; z. B. Taf. 13: B: 3, Hinweis d. Verf.).

In einigen südmährischen Fundkomplexen treten die Gefäße mit Rillenverzierung aller drei Varianten gemeinsam auf (Ľihovský 1982, 20), aber beim gegenwärtigen Forschungsstand muss mit einem früheren Vorkommen der dritten Variante gerechnet werden. Derart verzierte Keramik war nämlich in zwei in die mittlere Stufe der MHK datierten Fundkomplexen - im Brandgrab von Němčice und in einer Siedlungsgrube mit der Pithos-Bestattung eines Kindes in Olšany bei Prostějov enthalten_(Fojtik - Dočkalová 2007, 61, Taf. I: 2, 4; Fojtík 2015, 171, 193, Taf. 48: 2; 53: 4, 5). Auch in einer Siedlungsgrube von Brno-Černá Pole fand sich ein Fragment wohl einer Amphore mit solcher Verzierung; von K. Tihelka und V. Hank wurde diese Grube in die ältere Phase der MHK datiert (Tihelka - Hank 1949, 157; Salaš - Stuchlık 2013, Abb. 81: 3). Diese Variante der Rillenverzierung trifft beim Straninger Krug nicht zu.

Von J. Ř́hovský wurden im oben genannten Werk zahlreiche Krüge mit senkrechter Rillenverzierung der Varianten 1 und 2 abgebildet; von ihnen seien die bereits zitierten sechs Krü- ge aus dem Körpergrab der jüngeren Stufe der MHK von Brno-Maloměřice genannt (Ǩihouský 1982, 16, Taf. 2: 1, 4-7, 12). In der Slowakei findet der Krug von Straning eine gute Vergleichsmöglichkeit in einem rillenverzierten Krug im Grab 27 von Smolenice, allerdings wurde dieses Grab von V. Furmánek ungeachtet der späten chronologischen Stellung des Gefäßes aufgrund der Herzanhänger in die mittlere Stufe der MHK gesetzt (Dušek 1980, 347, Taf. IV: 9, 16-27; Furmánek 1980, 27). Da die zahlreichen Krüge mit Rillenverzierung der späten Stufe der MHK, die im Brandgrab 1 im Hügelgrab I von Bošovice $u$ a. mit einer Kugelkopfnadel mit geschwollenem Hals und einem Doppelkonus vergesellschaftet waren, des Öfteren auch noch Knubben auf der Schulter haben (Ǩíhouský 1982, Taf. 18: 12; 19-A: 2, 5), kann der Krug von Straning in die jüngere Stufe der MHK datiert werden.

Die Bedeutung des Grabes von Straning liegt also darin, dass durch sein Inventar die Lebensdauer fast aller aus verlässlich bronzedatierten Gräbern der älteren und der mittleren Stufe der mittleren Bronzezeit in Ostösterreich stammenden Typen der Herzanhänger, d. h. der Anhänger mit einfachem Mittelsteg, der Anhänger mit Mittelsteg und kurzen Fortsätzen und der Anhänger mit verzweigtem Mittelsteg und kurzen Fortsätzen bis in die jüngere Stufe dieser Kultur nachgewiesen ist, wobei dieses Typenspektrum auch noch um einen Anhänger selten vorkommenden Typs bereichert wird; einzig der Anhänger mit Mitteldorn aus dem Grab von Großhöflein (Willvonseder 1937, Taf. 24: 6) ist in Straning nicht vertreten. Durch das Inventar des Grabes 27 von Smolenice in der Westslowakei wird die Lebensdauer der Herzanhänger mit verzweigtem Mittelsteg und kurzen Fortsätzen bis in die jüngere Stufe der MHK bestätigt. Diese beiden Gräber sind nicht ohne Bedeutung für die Datierung der Grabfunde der MHK von Šatov in Südmähren (Furmánek 1973, 86, 126, Taf. 44: 3-5); die Herzanhänger 
mit Mittelsteg und kurzen Fortsätzen können, aber müssen nicht älter sein als der Anhänger mit einfachem Mittelsteg. Abschließend sei das Vorkommen von Herzanhängern auch noch in den urnenfelderzeitlichen Depotfunden in Oberösterreich erwähnt (Gruber 1999, 52); so stammt ein Anhänger mit Mittelsteg und kurzen Fortsätzen aus dem Depotfund von Linz-Freinberg (Willvonseder 1937, Taf. 43: 8; Erbach 1984, Taf. 58, o. Nr.).

Die Tote im Grab von Straning war, wie bereits erwähnt, in rechter Hockerlage bestattet. Die Bestattung in der Hockerlage ist im ganzen Verbreitungsgebiet der MHK - in Mittelböhmen, Südmähren, nördlichem Niederösterreich und der Westslowakei nachgewiesen (z. B. Beněs 1959, 5; Berg 1960, 42; Pichlerová 1976, 12; Hasenhündl 1989, 272; Stuchlik 1993, 277; 2006, 154; Fischbauer 2007, 246; Obenaus 2007, Abb. 10; Godiš - Haruštiak 2020, 54). Da spezielle Studien zum Bestattungsritus dieser Kultur noch ausständig sind, sei nur auf die diesbezügliche Situation im größten Gräberfeld der MHK - im birituellen Gräberfeld von Smolenice mit insgesamt 67 Gräbern - hingewiesen. Hier war nach M. Dušek die Totenlage infolge der sekundären Störungen nur bei 13 von insgesamt 25 Körpergräbern eruierbar. Die Verstorbenen waren in vier Gräbern in gestreckter Rückenlage (Gräber 15, 17, 53/72 und 67/73) und in 10 Gräbern in Hockerlage beigesetzt (Gräber 21, 25, 28, 42, 48, 77, 80, 132, 133/72 und $77 / 73$ ), d. h., dass die Bestattung in der Hockerlage überwiegt. Diese Zahlen vermitteln aber nur ein annäherndes Bild, da die Angaben und die bildliche Dokumentation zum Befund im Gegensatz zum Fundbestand in der Publikation des Gräberfeldes unzureichend sind. Die Seitenlage der Toten wurde nur bei vier von zehn Hockergräbern vermerkt, wobei eines von ihnen keine Funde enthielt und die Funde aus dem anderen nicht abgebildet wurden (Gräber 21/72, 25/72), aber die Angaben zu den restlichen zwei offenbar ungestörten Gräbern sind aufschlussreich. Das Bronzeinventar des Grabes 48/72, bestehend u. a. aus zwei Rollenkopfnadeln, spricht für ein Frauengrab; die Tote war in rechter Hockerlage bestattet, was der Lage einer 40-60-jährigen Frau im Grab 3 von Peigarten (Dušek 1980, 348, Taf. VI: 10, 11; Fischbauer 2007, 234, Abb. 4: 3; Novotny Spannagl-Steiner 2007, 256) und der Lage der Toten in Straning entspricht. Im Grab 77/73 von Smolenice fanden sich $u$. a. eine Nadel und sieben Bronzepfeilspitzen sowie eine Pfeilspitze aus Limnoquarzit, die die Annahme eines Männergrabes erlauben; der Tote war in linker Hockerlage beigesetzt (Dušek 1980, 351, Taf. VIII: 6, 11-19a). Das entspricht der Lage des Toten im Grab II im Hügelgrab 15 von Borotice, den S. Stuchlík für einen sich mit der Erzeugung von Bronzegegenständen befassenden Handwerker hält und $\mathrm{zu}$ dessen reicher Grabausstattung u. a. eine Nadel, ein Beil und eine Pfeilspitze gehörte; das Geschlecht und Alter des Toten konnten leider aufgrund der äußerst schlechten Erhaltungszustandes der Skelettreste nicht eruiert werden (Stuchlik 2006, 91, Abb. 87, 88; Dobisiková - Stuchlik 2014, 746). In linker Hockerlage war ein 20-30-jähriger Mann und in rechter Hockerlage ein 13-14-jähriges vermutliches Mädchen im Doppelgrab von Mikulov bestattet (Šabatová et al. 2020, 198, 200, 226, Abb. 5, 8A, 8B). Diese Angaben erwecken zwar den Eindruck einer geschlechtsmäßig gebundenen Bestattung, aber die Anzahl der zitierten Gräber ist zu gering, um derartige Schlüsse ziehen zu können, außerdem sind auch einige diesem Schema widersprechende Bestattungen bekannt. Auf der einen Seite sind zwei Gräber mit „weiblicher“ Ausstattung zu nennen; im Grab A von Dunajská Lužná war die Tote in linker Hockerlage, im Grab von Šamorín in der Rückenlage mit nach links angehockten Beinen bestattet (Pichlerová 1976, 12, Abb. 4, 7; Godiš - Haruštiak 2020, 54, Rekonstr. Abb. 7). Auf 
der anderen Seite ist das Grab 4 von Peigarten zu erwähnen, in welchem ein 35-45-jähriger Mann in rechter Hockerlage beigesetzt war (Fischbauer 2007, 234, Abb. 4: 4; Novotny - Spannagl-Steiner 2007, 254).

Diese knappen Beobachtungen zur Bestattung in der Hockerlage sollen daher beim gegenwärtigen Forschungsstand nur als ein Ansporn für eingehende Untersuchungen der vielfältigen Bestattungsweise der MHK verstanden werden, einer birituellen Kultur, die ihre Toten sowohl auf Hügelgräberfeldern als auch auf flachen Gräberfeldern bestattete und die sowohl die Körperbestattung als auch die Brandbestattung auf mannigfaltige Wei-

\section{Literatur}

Bartik, J. - Farkaš, Z. - Jelínek, P. 2019: Bronzehortfunde aus den Kleinen Karpaten und ihren Vorgebirgsgebieten. In: I. Bazovský - G. Březinová (ed.), Ludia a hory - archeologická perspektíva. Interakcie ludských spoločenstiev horských a podhorských oblastí západného Slovenska. Zborník SNM, Supplementa 12. Bratislava, 15-102.

Beněs, A, 1959: K problémům mohylové kultury doby bronzové ve středních Čechách. Sborník Národního muzea v Praze XIII Nr. 1-2.

Benkovsky-Pivovarová, Z. 1976: Zur Genese der Urnenbestattung in der Lausitzer Kultur Mährens, der Slowakei und Südpolens. Jahresbericht des Instituts für Vorgeschichte der Universität Frankfurt a. M. 1975. München, 61-72.

Benkovsky-Pivovarová, Z. 1985: Das Bronzeinventar des mittelbronzezeitlichen Gräberfeldes von Pitten, Niederösterreich. Mitteilungen der Prähistorischen Kommission der ÖAW XXI/XXII, 19821985, 23-126.

Berg, F. 1960: Mittelbronzezeitliche Grabfunde aus Maiersch und Theras, N. Ö. Archaeologia Austriaca 27, 21-51.

Dobisiková, M. - Stuchlik, S. 2014: Mohylník v Boroticích z pohledu antropologie. In: J. Čižmářo- se ausübte; bei der Körperbestattung ist die gestreckte Rückenlage und die Hockerlage belegt, bei der Brandbestattung sind Urnengräber, Grubengräber und Brandschüttungsgräber nachgewiesen (Stuchlik 2006, 154); beachtenswert ist die hohe Anzahl der Urnengräber in Mähren (Kartierung: Benkovsky-Pivovarová 1976, Abb. 1). Die Untersuchungen zur Bestattungsweise der MHK sind allerdings nicht nur an die Veröffentlichung der bis jetzt unpublizierten Gräber und Gräberfelder dieser Kultur, sondern in einigen Fällen auch an die erneute Vorlage der unzureichend veröffentlichten Quellen, wie z. B. des Gräberfeldes von Smolenice, gebunden.

vá - N. Venclová - G. Březinová (eds.), Moravské křižovatky. Střední Podunají mezi pravěkem a historií. Brno, 739-754.

Dušek, M. 1980: Pohrebisko stredodunajskej mohylovej kultúry v Smoleniciach. Slovenská archeológia XXVIII, 341-382.

Erbach, M. zu 1984: Die spätbronze- und urnenfelderzeitlichen Funde aus Linz und Oberösterreich. Linzer archäologische Forschungen, Band 14. Linz.

Fischbauer, S. 2007: Ein mittelbronzezeitliches Gräberfeld in Peigarten, Niederösterreich. Fundberichte aus Österreich 45, 2006, 233-256.

Fojtik, P. 2015: Mohylová kultura střední doby bronzové na Prostějovsku I. Pravěk Supplementum 30, Brno.

Fojtik, P. - Dockalová, M. 2007: Středobronzový pohřeb v nádobě z Olšan u Prostějova. Študijné zvesti Archeologického ústavu SAV 42, 57-69.

Furmánek 1973: Bronzová industrie středodunajské mohylové kultury na Moravě. Slovenská archeológia XXI-1, 25-145.

Furmánek, V. 1980: Die Anhänger in der Slowakei. Prähistorische Bronzefunde XI-3, München.

Godiš, J. - Haruštiak, J. 2020: Unikátny ženský hrob mohylovej kultúry v Šamoríne. In: V. Mitáš - O. Oždáni (ed.), Doba popolnicových polí a doba 
halštatská. Zborník príspevkov z XV. medzinárodnej konferencie Doba popolnicových polí a doba halštatská, Smolenice 15.-19. októbra 2018. Archaeologica Slovaca Monographiae, Communicationes XXV, Nitra 2020, 53-75.

Gruber, K. H. 1999: Die mittelbronzezeitlichen Grabfunde aus Linz und Oberösterreich. Linzer archäologische Forschungen 28, 1999.

Hampl, F. - Kerchler, H.- Benkovsky-Pivovarová, Z. 1981: Das mittelbronzezeitliche Gräberfeld von Pitten in Niederösterreich. Mitteilungen der Prähistorischen Kommission der ÖAW XIX-XX, 1978-1981.

Hasenhündl, G. 1989: KG Hollabrunn, SG Hollabrunn, VB Hollabrunn. Fundberichte aus Österreich 27, 1988, 272.

Hänsel, B., 1968: Beiträge zur Chronologie der mittleren Bronzezeit im Karpatenbecken. Beiträge zur ur- und frühgeschichtlichen Archäologie des Mittelmeer-Kulturraumes 7, Bonn.

Hlas, J. - Stuchlik, S. - Šin, L. 2017: Únětické pohřebiště v Opavě-Vávrovicích. Památky archeologické CVIII, 39-88.

Koschik, H. 1981: Die Bronzezeit im südwestlichen Oberbayern. Materialhefte zur Bayerischen Vorgeschichte 50, 1981.

Lauermann, E. 1991: Neue mittelbronzezeitliche Funde aus dem Weinviertel, NÖ. Fundberichte aus Österreich 29, 1990, 35-43.

Maurer, H. 1989: Einführung in die Vor- und Frühgeschichte des niederösterreichischen Waldviertels. In: H. Maurer - F. Stojaspal - R. Puschnik - H. Lederer, Aus der Vorzeit des niederösterreichischen Waldviertels. Mannus-Bibliothek XXXII, 26-76.

Mozsolics, A. 1967: Bronzefunde des Karpatenbeckens. Depotfundhorizonte von Hajdúsámson und Kosziderpadlás, Budapest.

Müller-Karpe, H. 2003: Zur religiösen Symbolik von bronzezeitlichem Trachtschmuck aus Mitteleuropa. Anodos, Studies of the Ancient World 3, 145-154.

Novotná, M. 1980: Die Nadeln in der Slowakei. Prähistorische Bronzefunde XIII-5, München.

Novotny, F. - Spannagl-Steiner, M. 2007: Anthropologischer Befund der menschlichen Skelettreste. In: S. Fischbauer 2007, 252-254.
Obenaus, M. 2007: Archäologische Untersuchungen im ehemaligen Minoritenkloster in Stein, Stadt Krems an der Donau. Fundberichte aus Österreich 45, 2006, 569-581.

Pichlerová, M. 1976: Pohrebisko stredodunajskej mohylovej kultúry a iné nálezy z Dunajskej Lužnej, okres Bratislava-vidiek. Zborník SNM LXX, História 16, 5-29.

Řihouský, J. 1982: Základy středodunajských popelnicových polí na Moravě. Studie AÚČSAV X, Brno. Salaš, M. - Stuchlik, S. 2013: Civilizace doby bronzové. In: R. Procházka (red.), Dějiny Brna 1. Od pravěku k ranému středoveku, Brno, 228-318.

Schäffer, F. 1957: Fundbericht des Krahuletz-Museums der Stadt Eggenburg (Nr. 152), betreffend ein Frauengrab der Mittelbronzezeit aus Straning. Nachrichtenblatt für die österreichische Ur- und Frühgeschichtsforschung VI, 1-3.

Schumacher-Matthäus, G. 1985: Studien zu bronzezeitlichen Schmucktrachten im Karpatenbecken. Ein Beitrag zur Deutung der Hortfunde im Karpatenbecken. Marburger Studien zur Vor- und Frühgeschichte Mainz am Rhein.

Stuchlik, S. 1993: Středodunajská mohylová kultura. In: V. Podborský s kolektivem, Pravěké dějiny Moravy. Vlastivěda moravská. Země a lid. NŘ, sv. 3, Brno, 272-286.

Stuchlik, S. 2006: Borotice. Mohylové pohřebiště z doby bronzové. Spisy Archeologického ústavu 30, Brno.

Šabatová, K. - Parma, D. - Trampota, F. - Jarošová, I. - Kañáková Hladíková, L. - Přichystal, A. - Vargová, L. - Vymazalová, K. 2020: Hrob s kamennou konstrukcí ze střední doby bronzové z Mikulova. Archeologické rozhledy LXXII, 2, 194-237.

Tihelka, K. - Hank, V. 1949: Sídlište únětické a středodunajské mohylové kultury v Brně-Černých Polích. Časopis Moravského muzea XXXIV, 138157.

Wels-Weyrauch, U. 1991: Die Anhänger in Südbayern. Prähistorische Bronzefunde XI-5, Stuttgart.

Willvonseder, K. 1937: Die mittlere Bronzezeit in Österreich, Wien-Leipzig. 


\section{On a grave of the Middle Danube Tumulus Culture from Straning, Lower Austria}

In 1957, F. Schäffer, the then custodian of the Museum in Eggenburg, has published the finds from a recently disturbed inhumation grave at Straning in the district of Horn (Schäffer 1957, 1-3). The only preserved parts of the skeleton were skull and bones of the right arm and right leg in a contracted position on the right side. Since the skull "looks to the east", the buried individual was supposedly oriented approximately from the south to the north; according to the preserved skeletal remains and jewellery, F. Schäffer identified the individual as a young woman.

The preserved parts of funerary equipment comprise a pin, five heart-shaped pendants, seven tutuli and a jug (Fig. 1). The jug rested at the vertex of the skull, the position of the pin and five tutuli could not be determined after the find was announced. Heart-shaped pendants were found in the neck area and two tutuli rested at the knee on the thigh bone and calf bone, so that F. Schäffer supposed them to belong to clothing accessories. The bones of the right forearm were coloured by bronze patina.

The pin and the tutuli cannot be used as a dating criterion due to their long-term occurrence. Heart-shaped pendants represent a typical female ornament, which was mainly used as a component of necklaces (Schumacher-Matthäus 1985, 105). The necklace in the grave from Straning consisted of four types of pendants (type 1-4), of which the type 2 occurred in two variants. Because the jug is the most important dating factor in the grave, the analogies to each type of pendants are only represented by pendants from reliably dated graves in East Austria. In addition, the terms which are used for these types of pendants in literature are mentioned together with their dating. Fluted decoration on pottery in the late and final phases of the Middle Danube Tumulus Culture (hereinafter MDTC) was treated in detail by J. Ř́hovský, who distinguished three variants of fluting (Řihovský 1982, 19 f.). Several assemblages of finds from Moravia contained all three variants, but in Ř́hovský's variant 3, i.e. flutes separated from one another by free space, we must take into account the finds from Brno-Černá Pole, Němčice and Olšany and suppose a much earlier occurrence of this variant in comparison to variants 1 and 2, i.e. flutes that form ribs and "classical" flat flutes (Tihelka - Hank 1949, 157; Fojtik - Dočkalová 2007, 61, tab. I: 2, 4; Salaš - Stuchlik 2013, obr. 81: 3; Fojtik 2015, 171, 193, tab. 48: 2; 53 $4,5)$. The jug from Straning, decorated with "classical" fluting, can be dated on the basis of analogous finds to the later phase of MDTC (e.g. Řihovsky 1982, 16, tab. 2: 1, 4-7, 12). It is true that similar jugs also occur in the final phase of this culture, but these late forms often have knobs under the neck/belly transition (Řihovský 1982, tab. 18: 12; 19-A: 5).

The pendants from Straning confirm the occurrence of almost all types of heart-shaped pendants from reliably dated graves of the early and middle phases of the Middle Bronze Age on the sites of Großhöflein, Hollabrunn and Pitten in East Austria until the late phase of the Middle Bronze Age and enrich them with another rare type; only the pendant with central spike from a grave in Großhöflein is not present at Straning (Willvonseder 1937, Taf. 24: 2-4; Hampl - Kerchler - Benkovsky-Pivovarová 1981, Taf. 203: 6-8; Benkovsky-Pivovarová 1985, 72; Lauermann 1990, 37, Taf. 4: 4-26). The presence of pendants of type Dunajská Streda in Grave 27 at Smolenice in association with a jug with vertical fluting confirms their occurrence until the late phase of MDTC (Dušek 1980, tab. IV: 9; Furmánek 1980, 29 f.).

Contracted burials in MDTC are evidenced in the whole distribution territory of this culture. In the largest cemetery of this culture in Smolenice, contracted burials outnumbered the burials in stretched supine position. The data on the position of individuals buried in this cemetery in many cases do not suffice for a thorough analysis of burial rites. However, the given data on contracted burials and the bronze artefacts from graves $48 / 72$ and $77 / 73$ indicate that men were buried on the left side and women on the right side (Dušek 1980, 348, 351, tab. VI: 10, 11; VIII: 6, 11-19a). This assumption is in 
accordance with anthropological analysis, position of buried individuals or with funerary equipment in several graves in South Moravia and Lower Austria (Borotice, Grave 15-II: Stuchlik 2006, 91, obr. 87, 88; Mikulov: Šabatová et al. 2020, 198, 200, 226, obr. 5, 8A, 8B; Peigarten, Grave 39: Fischbauer 2007, 234, Abb. 4: 3; Novotny - Spannagel-Steiner 2007, 256), but the number of these graves is too low to draw any generally valid conclusions. Moreover, these detections are not in compliance with the position of buried individuals in several other graves in Slovakia and Lower Austria (Dunajská Lužná, Grave A:
Pichlerová 1976, 12, obr. 4, 7; Šamorín: Godiš - Ha ruštiak 2020, 54, reconstruction obr. 7; Peigarten, Grave 4: Fischbauer 2007, 246, Abb. 4: 4; Novotny - Spannagl-Steiner 2007, 254). MDTC was a biritual culture, whose people buried their dead both in barrows and in flat cemeteries in multiple ways, including both inhumation and cremation (Stuchlik $2006,154)$. The precondition for an intensive study of the burial rites of MDTC is not only the publication of all graves and cemeteries, but in some cases also a new processing of insufficiently published materials, e.g. the cemetery at Smolenice.

\section{Dr. Zoja Benkovsky-Pivovarová}

- Donaustraße 89/5

A - 2344 Maria Enzersdorf-Südstadt

zoja.benkovsky@gmail.com 